\title{
Effects of estrogen on glutathione and catalase levels in human erythrocyte during menstrual cycle
}

\author{
SHENG-HUANG CHANG $^{1 *}$, CHIEH-HSIN CHANG $^{1 *}$, MU-CHUN YANG $^{2}$, WEN-TUNG HSU $^{3}$, CHIA-YING HSIEH $^{1}$, \\ YA-TING HUNG ${ }^{1}$, WAN-LING SU ${ }^{4}$, JIUAN-JEN SHIU ${ }^{5}, \mathrm{CHIH}^{-Y A N G ~ H U A N G}{ }^{6-8}$ and JER-YUH LIU ${ }^{5,9}$ \\ ${ }^{1}$ Department of Laboratory, Tsaotun Psychiatric Center, Ministry of Health and Welfare, Caotun, Nantou 542; \\ ${ }^{2}$ Department of Medicine, China Medical University, Taichung 40402; ${ }^{3}$ Department of Laboratory, \\ Taichung Armed Force General Hospital, Taichung 41152; ${ }^{4}$ Institute of Biochemistry and Biotechnology, \\ Chung Shan Medical University, Taichung 40201; ${ }^{5}$ Center for Molecular Medicine, China Medical University, \\ Taichung 40402; ${ }^{6}$ Graduate Institute of Chinese Medical Science, China Medical University, Taichung; \\ ${ }^{7}$ Graduate Institute of Basic Medical Science, China Medical University, Taichung 40402; \\ ${ }^{8}$ Department of Health and Nutrition Biotechnology, Asia University, Taichung; \\ ${ }^{9}$ Graduate Institute of Cancer Biology, China Medical University, Taichung 40402, Taiwan, R.O.C.
}

Received November 4, 2014; Accepted December 19, 2014

DOI: $10.3892 /$ br. 2014.412

\begin{abstract}
The present study evaluated the effects of physiological serum estrogen during the menstrual cycle on glutathione (GSH) and catalase activities. The sample included 43 healthy females between the ages of 22 and 51 years. The subjects were divided into two groups according to the stage of the menstrual cycle. Group A consisted of 16 samples extracted between days 10 and 20 from the first day of menstruation when estrogen levels were considered to be at their highest. Group B consisted of 27 samples extracted during other times of the estimated 30 days of menstruation. Data showed that the estrogen level in group A $(184 \pm 106 \mathrm{pg} / \mathrm{ml})$ was higher than that in group $\mathrm{B}(105 \pm 56 \mathrm{pg} / \mathrm{ml})(\mathrm{P}<0.01)$. The GSH and catalase levels in group A $(4.4 \pm 2.3 \mu \mathrm{g} / \mathrm{mg}$ and $210 \pm 72 \mathrm{IU} / \mathrm{mg}$, respectively) were also significantly higher compared to the levels in group B $(3.2 \pm 1.8 \mu \mathrm{g} / \mathrm{mg}$ and $168 \pm 62 \mathrm{IU} / \mathrm{mg}$, respectively) $(\mathrm{P}<0.05)$. Spearman's rank correlation showed that the expression of catalase in red blood cells significantly correlated with serum estrogen level but not with GSH. However, the changes in estrogen plasma levels, erythrocyte GSH level and catalase activity suggested that the consumption of GSH and catalase in erythrocyte during the menstrual cycle may be associated with the level of estrogen present in the bloodstream.
\end{abstract}

Correspondence to: Dr Jer-Yuh Liu, Graduate Institute of Cancer Biology, College of Medical, China Medical University, 91 Hsueh-Shih Road, Taichung 40402, Taiwan, R.O.C.

E-mail: jyl@mail.cmu.edu.tw

${ }^{*}$ Contributed equaly

Key words: estrogen, glutathione, catalase, menstrual cycle

\section{Introduction}

Antioxidant defenses help to prevent free radical-mediated injury $(1,2)$. These substances, including enzymatic (catalase, superoxide dismutases, glutathione peroxidase and glutathione reductase) and non-enzymatic [vitamin E, vitamin C, glutathione (GSH) and uric acid] categories, protect an organism from oxygen free radicals (3). Red blood cells (RBCs) are the main source of antioxidants; in vitro and in vivo tests have been performed to demonstrate the importance of RBC antioxidant enzymes in protecting target cells from reactive oxygen species (ROS) $(4,5)$. Therefore, the amount of antioxidants in RBC is a suitable marker for evaluating chronic ROS exposure.

GSH is the most abundant thiol in cells and acts as a major antioxidant in addition to its other biological functions in RBC (6). Catalase is an important antioxidant produced by RBC; thus, catalase levels can indicate ROS levels (7). However, limited information is available on the effects of physiological sex steroid changes during the menstrual cycle on the GSH and catalase in RBC in a population of healthy normomenorrhoic females. Therefore, the present study evaluated the effects of physiological sex steroid changes during the menstrual cycle on GSH and catalase activities. Females with regular menstrual cycles were selected to be part of the sample and the changes in the GSH and catalase levels during the menstrual cycle were measured. The resulting correlation between estrogen and antioxidant systems can be used for analyses and discussion.

\section{Materials and methods}

Subjects. The study population consisted of 43 healthy female volunteers (age range, 22-51 years; mean age, 36 years), with a history of regular menstrual cycles lasting from 28 to 30 days. Their health status was confirmed on the basis of their medical history, as well as by physical and routine laboratory 
examinations. Body weight was normal and all the subjects were on a standard balanced diet. None of the subjects smoked or had a history of intensive exercise. None had used oral contraceptives for at least one year before the study or any other type of medication during the previous four months. No drugs were taken during the study period. Informed consent was obtained from all the subjects prior to participation in the study. The study protocol was approved by the Tsaotun Psychiatric Center, Ministry of Health and Welfare, Taiwan.

Sample collection. After overnight fasting, blood samples were collected by vein puncture and stored on ice. Erythrocytes were immediately washed two times in phosphate-buffered saline. Erythrocyte hemodialysates for the estimation of catalase enzyme activity and GSH concentration were prepared by centrifugation of heparinized blood $(25,910 \mathrm{x} \mathrm{g}$ for $10 \mathrm{sec}$ at $4^{\circ} \mathrm{C}$ ), deprived of buffy coats and stored at $-20^{\circ} \mathrm{C}$.

Catalase activity. The determination of catalase was assayed as described (8). The reaction mixture $(1 \mathrm{ml})$ contained $50 \mu \mathrm{l}$ Tris-EDTA (pH 8.0) and $900 \mu 120 \mathrm{mmol} / 1 \mathrm{H}_{2} \mathrm{O}_{2}$ heated to $37^{\circ} \mathrm{C}$ for $10 \mathrm{~min}$, after which $20 \mu \mathrm{l}$ erythrocyte lysate was added. The optical density value at $240 \mathrm{~nm}$ was detected for $1 \mathrm{~min}$ at $25^{\circ} \mathrm{C}$. The catalase activity was expressed in $\mathrm{IU} / \mathrm{mg}$ protein ( $\mu$ moles of $20 \mathrm{mmol} / 1 \mathrm{H}_{2} \mathrm{O}_{2}$ consumed/min/mg protein).

Erythrocyte GSH level. Erythrocyte GSH was measured using a spectrophotometric method of the form of reduced GSH (9). 5,5'-Dithiobis-2-nitrobenzoic acid is a disulfide chromogen that is readily reduced by sulfhydryl compound to an intensely yellow compound. The absorbance of the reduced compound was measured at $412 \mathrm{~nm}$ as directly proportional to GSH concentration.

Protein concentrations. Protein concentrations were quantified using the Bradford protein assay method.

Estrogen measurement. The serum estrogen concentration was determined by radioimmunoassay (Diagnostic Products Corporation, Los Angeles, CA, USA).

Statistical analysis. Each specimen was analyzed twice with the use of Student's t-test analysis software. $\mathrm{P}<0.05$ was considered to indicate a significant difference and $\mathrm{P}<0.01$ was considered to represent an extremely significant difference. Pearson's or Spearman's analysis was used in the correlation analysis.

\section{Results}

GSH levels in erythrocytes during the menstrual cycle. The sample subjects were divided into two groups according to the number of days the subjects were into the menstrual cycle. Group A (16 samples) consisted of samples from females that were within 10-20 days of their menstruation. Group B (27 samples) consisted of females that were 1-9 and 21-30 days into their menstruation. The average estrogen level in group $\mathrm{A}$ $(184 \pm 106 \mathrm{pg} / \mathrm{ml})$ was higher than that in group B $(105 \pm 56 \mathrm{pg} / \mathrm{ml})$ $(\mathrm{P}<0.01)$. The GSH levels in group A $(4.4 \pm 2.3 \mu \mathrm{g} / \mathrm{mg})$ were also significantly higher compared to group B $(3.2 \pm 1.8 \mu \mathrm{g} / \mathrm{mg})$

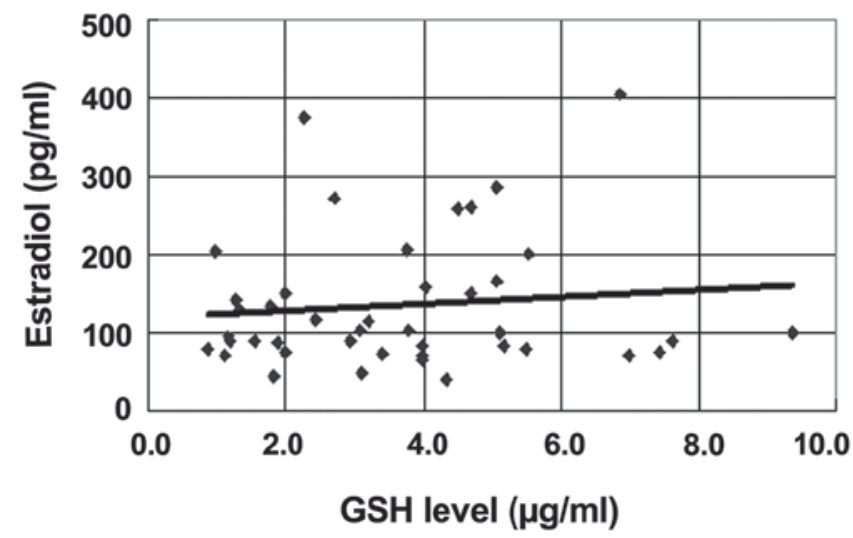

Figure 1. Correlation between the serum estradiol and erythrocyte glutathione $(\mathrm{GSH})$ levels in regular menstrual females. $\mathrm{P}=0.33$ indicates an insignificant correlation.

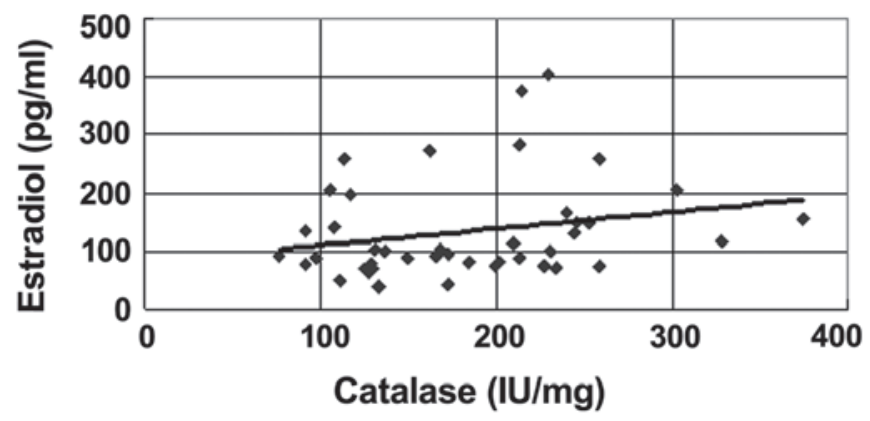

Figure 2. Correlation between the serum estradiol level and erythrocyte catalase activity in regular menstrual females. $\mathrm{P}=0.04(\mathrm{P}<0.05)$ indicates a significant correlation.

$(\mathrm{P}<0.05)$. However, when Spearman's rank correlation analysis was performed, no significant correlation $(\mathrm{P}=0.32)$ was found between the estrogen levels in the blood and the GSH expression in the RBC (Fig. 1).

Catalase activity in the RBC during the menstrual cycle. The average level of catalase in group A $(210 \pm 72 \mathrm{IU} / \mathrm{mg})$ was significantly higher than that in group B $(168 \pm 62 \mathrm{IU} / \mathrm{mg})(\mathrm{P}<0.05)$. In the Spearman's rank correlation analysis, a significant correlation $(\mathrm{P}=0.04)$ was found between the estrogen levels in the blood and the catalase expression in the RBC (Fig. 2).

\section{Discussion}

Oxidative stress is responsible for aging and coronary heart diseases $(10,11)$. Rapid induction of intracellular GSH synthesis occurs in response to various oxidative stress parameters, which is a critical determinant of cellular tolerance to oxidative stress (12). Damy et al (13) reported that cardiac and systemic GSH deficiency is associated with the functional status and structural cardiac abnormalities of patients with cardiac diseases. As a result, GSH serves an important function in fighting oxidative damage and helping prevent heart diseases. Additionally, the GSH level is consistently increased following various types of treatments and in different situations, such as amenorrhea, post-menopause or treatments with 
oral contraceptive drugs (14-17). In the present study, blood samples were collected from females who were not taking contraceptive drugs, between ages 22 and 51 years and have regular menstrual cycles. The antioxidant levels of the samples were determined on the same day to avoid hemolysis. In a regular menstrual cycle, the GSH level increased when the estrogen concentration was significantly increased.

An antioxidant-like catalase is also inversely associated with coronary heart disease (18). However, Yang et al (19) reported that high levels of catalase activity were associated with a low risk of coronary heart diseases in females with diabetes but not in those without diabetes. Pre-menopausal females have a lower risk of heart diseases compared to males, but the risk becomes the same post-menopause (20). Estrogen levels exist in a high concentration pre-menopause, which decreases oxidative stress in the cardiovascular system. In the present data, estrogen levels increased and decreased according to the menstrual cycle and the catalase level shifted according to the cycle. This process may protect females from cardiovascular diseases.

The effect of estrogen on catalase in the condition of elevation in previous studies differed from the results of the present study. Massafra et al $(21,22)$ reported that a significant positive correlation existed between physiological estrogen and antioxidant expression during the menstrual cycle, but no significant changes occurred in catalase. However, females in different situations, such as multiple blood sampling, which thus have different effects, may take different mechanism routes. Therefore, the present study underlined a significant correlation between estrogen and catalase/GSH and suggested that estradiol upregulated the expression of the antioxidants, which may also be mediated through the same mechanism. Certain unknown factors involved in the whole pathway should be further investigated.

\section{Acknowledgements}

The present study was supported by the grant (no. 9818) from Tsaotun Psychiatric Center of Ministry of Health and Welfare, Taiwan.

\section{References}

1. Southorn PA and Powis G: Free radicals in medicine. I. Chemical nature and biologic reactions. Mayo Clin Proc 63: 381-389, 1988.

2. Southorn PA and Powis G: Free radicals in medicine. II. Involvement in human disease. Mayo Clin Proc 63: 390-408, 1988

3. Sakac V and Sakac M: Free oxygen radiacals and kidney diseases - part I. Med Pregl 53: 463-474, 2000 (In Croatian).
4. Agar NS, Sadrzadeh SM, Hallaway PE, et al: Erythrocyte catalase. A somatic oxidant defense? J Clin Invest 77: 319-321, 1986.

5. van Asbeck BS, Hoidal J, Vercellotti GM, et al: Protection against lethal hyperoxia by tracheal insufflation of erythrocytes: role of red cell glutathione. Science 227: 756-759, 1985.

6. Dabrosin C and Ollinger K: Variability of glutathione during the menstrual cycle-due to estrogen effects on hepatocytes? Free Radic Biol Med 36: 145-151, 2004.

7. Nishikawa M, Hashida M and Takakura Y: Catalase delivery for inhibiting ROS-mediated tissue injury and tumor metastasis. Adv Drug Deliv Rev 61: 319-326, 2009.

8. Kolberg C, Horst A, Kolberg A, et al: Effects of high-velocity, low-amplitude manipulation on catalase activity in men with neck pain. J Manipulative Physiol Ther 33: 300-307, 2010.

9. Owens CW and Belcher RV: A colorimetric micro-method for the determination of glutathione. Biochem J 94: 705-711, 1965.

10. Cencioni C, Spallotta F, Martelli F, et al: Oxidative stress and epigenetic regulation in ageing and age-related diseases. Int $\mathrm{J}$ Mol Sci 14: 17643-17663, 2013.

11. Halliwell B: The role of oxygen radicals in human disease, with particular reference to the vascular system. Haemostasis 23 (Suppl 1): 118-126, 1993.

12. Rahman I, Antonicelli F and MacNee W: Molecular mechanism of the regulation of glutathione synthesis by tumor necrosis factor-alpha and dexamethasone in human alveolar epithelial cells. J Biol Chem 274: 5088-5096, 1999.

13. Damy T, Kirsch M, Khouzami L, et al: Glutathione deficiency in cardiac patients is related to the functional status and structural cardiac abnormalities. PLoS One 4: e4871, 2009.

14. Massafra C, Buonocore G, Berni S, et al: Antioxidant erythrocyte enzyme activities during oral contraception. Contraception 47: 590-596, 1993.

15. Massafra C, Buonocore G, Gioia D, et al: Changes in the erythrocyte antioxidant enzyme system during transdermal estradiol therapy for secondary amenorrhea. Gynecol Endocrinol 10: 155-158, 1996.

16. Massafra C, Buonocore G, Gioia D, et al: Effects of estradiol and medroxyprogesterone-acetate treatment on erythrocyte antioxidant enzyme activities and malondialdehyde plasma levels in amenorrhoic women. J Clin Endocrinol Metab 82: 173-175, 1997.

17. Bednarek-Tupikowska G, Tworowska U, Jedrychowska I, et al: Effects of oestradiol and oestroprogestin on erythrocyte antioxidative enzyme system activity in postmenopausal women. Clin Endocrinol (Oxf) 64: 463-468, 2006.

18. Flores-Mateo G,Carrillo-Santisteve P,Elosua R, et al: Antioxidant enzyme activity and coronary heart disease: meta-analyses of observational studies. Am J Epidemiol 170: 135-147, 2009.

19. Yang S, Jensen MK, Rimm EB, et al: Erythrocyte superoxide dismutase, glutathione peroxidase and catalase activities and risk of coronary heart disease in generally healthy women: a prospective study. Am J Epidemiol 180: 901-908, 2014.

20. Yang XP and Reckelhoff JF: Estrogen, hormonal replacement therapy and cardiovascular disease. Curr Opin Nephrol Hypertens 20: 133-138, 2011.

21. Massafra C, De Felice C, Gioia D, et al: Variations in erythrocyte antioxidant glutathione peroxidase activity during the menstrual cycle. Clin Endocrinol (Oxf) 49: 63-67, 1998.

22. Massafra C, Gioia D, De Felice C, et al: Effects of estrogens and androgens on erythrocyte antioxidant superoxide dismutase, catalase and glutathione peroxidase activities during the menstrual cycle. J Endocrinol 167: 447-452, 2000. 